\title{
Single-Dimensional Human Brain Signals for Two-Dimensional Economic Choice Options
}

\author{
${ }^{\circledR}$ Leo Chi U Seak, ${ }^{\star}$ Konstantin Volkmann, ${ }^{\star}$ Alexandre Pastor-Bernier, Fabian Grabenhorst, and \\ (1)Wolfram Schultz \\ Department of Physiology, Development and Neuroscience, University of Cambridge, Cambridge CB2 3DY, United Kingdom
}

Rewarding choice options typically contain multiple components, but neural signals in single brain voxels are scalar and primarily vary up or down. In a previous study, we had designed reward bundles that contained the same two milkshakes with independently set amounts; we had used psychophysics and rigorous economic concepts to estimate two-dimensional choice indifference curves (ICs) that represented revealed stochastic preferences for these bundles in a systematic, integrated manner. All bundles on the same ICs were equally revealed preferred (and thus had same utility, as inferred from choice indifference); bundles on higher ICs (higher utility) were preferred to bundles on lower ICs (lower utility). In the current study, we used the established behavior for testing with functional magnetic resonance imaging (fMRI). We now demonstrate neural responses in reward-related brain structures of human female and male participants, including striatum, midbrain, and medial orbitofrontal cortex (mid-OFC) that followed the characteristic pattern of ICs: similar responses along ICs (same utility despite different bundle composition), but monotonic change across ICs (different utility). Thus, these brain structures integrated multiple reward components into a scalar signal, well beyond the known subjective value coding of single-component rewards.

Key words: bundles; decision-making; fMRI; revealed preference; reward; stochastic choice

Significance Statement

Rewards have several components, like the taste and size of an apple, but it is unclear how each component contributes to the overall value of the reward. While choice indifference curves (ICs) of economic theory provide behavioral approaches to this question, it is unclear whether brain responses capture the preference and utility integrated from multiple components. We report activations in striatum, midbrain, and orbitofrontal cortex (OFC) that follow choice ICs representing behavioral preferences over and above variations of individual reward components. In addition, the concept-driven approach encourages future studies on natural, multicomponent rewards that are prone to irrational choice of normal and brain-damaged individuals.

\section{Introduction}

In daily life, we choose between options that have multiple components. In a restaurant, we can get, for the same price, a small but tasty steak or a larger but less tasty steak. In choosing the

Received June 19, 2020; revised Oct. 28, 2020; accepted Nov. 3, 2020.

Author contributions: K.V., A.P.-B., F.G., and W.S. designed research; K.V. performed research; L.C.U.S., K.V., A.P.-B., and F.G. analyzed data; L.C.U.S. and W.S. wrote the paper.

This work was supported by Wellcome Trust Grants WT 095495 and WT 204811. We thank Charles R. Plott for discussions and conceptual support, Steve Edgley for help and logistic support, Simone Ferrari-Toniolo for comments on experimental economics, Jae-Chang Kim and Putu Agus Khorisantono for suggestions on fMRI analysis, and Arkadiusz Stasiak for computer support.

*L.C.U.S. and K.V. contributed equally to this work.

The authors declare no competing financial interests.

Correspondence should be addressed to Wolfram Schultz at Wolfram.Schultz@Protonmail.com.

https://doi.org/10.1523/JNEUROSCI.1555-20.2020

Copyright $\odot 2021$ Seak, Volkmann et al.

This is an open-access article distributed under the terms of the Creative Commons Attribution 4.0 International license, which permits unrestricted use, distribution and reproduction in any medium provided that the original work is properly attributed. latter, we give up some taste for more meat. Or the components can be distinct objects, like a meal with small lasagna and big salad, or a meal with large lasagna and small salad; in choosing the latter, we give up some salad for more lasagna. In both cases, our preference for an option (steak or meal) is based on more than one component. To understand such choices, we need to know whether the value integrated from different components can be represented by scalar measures of preferences and their neuronal processes.

Functional magnetic resonance imaging (fMRI) studies investigated choices between bundles with multiple-components. Several brain regions are involved in such choices, including striatum (Hunt et al., 2014), frontal cortex (Hunt et al., 2014; Busemeyer et al., 2019; Kurtz-David et al., 2019), cingulate cortex (Fujiwara et al., 2009; Busemeyer et al., 2019; Kurtz-David et al., 2019), and insula (Busemeyer et al., 2019). One study showed encoding of values of gift cards that contained an amount component and a quality component (de Berker et al., 2019), other 
studies investigated irrational choices with monetary-gamble components (Kurtz-David et al., 2019) and addressed irrational attraction and decoy effects (Chau et al., 2014; Chung et al., 2017; Gluth et al., 2017). Whereas these studies demonstrated neural signals for multicomponent rewards, they did not specifically investigate whether the signals captured the reward value integrated from multidimensional vectorial choice options. To resolve the issue would require to study how the increase of one component compensates for the decrease of the other component without changing the preference, and how such a trade-off is represented in scalar neural signals.

This trade-off mechanism constitutes the heart of indifference curves (ICs) underlying revealed preference theory (Samuelson, 1938). Each two-component choice option is graphically represented at a specific $x-y$ coordinate of a two-dimensional plot (Mas-Colell et al., 1995). All bundles that are equally preferred to each other (choice indifferent, indicating same utility despite different bundle composition) are located on the same IC regardless of underlying variation in bundle composition. Preferred bundles are located on higher ICs (farther away from the origin, higher utility). This scheme is widely used for conceptualizing economic preferences in economics textbooks, consumer choice (Simonson, 1989; Tversky and Simonson, 1993; Rieskamp et al., 2006), animal choice (Kagel et al., 1975; Pastor-Bernier et al., 2017), and neuronal reward signals in animals (Pastor-Bernier et al., 2019). The preference scheme has been extended to stochastic choice (McFadden and Richter, 1990; McFadden, 2005), which is helpful for multitrial statistical analyses of human brain responses. Thus, the question for the current study arises: would human blood oxygen level-dependent (BOLD) signals follow the characteristics of ICs that define the emergence of scalar measures from vectorial bundles?

We investigated scalar BOLD signals for two-component milkshakes with sugar and fat components that elicit subjective valuations and neural reward signals (Grabenhorst et al., 2010; Zangemeister et al., 2016). We used three revealed preference levels (three ICs, different utility), each estimated from five equally preferred bundles [indifference points (IPs) located on same IC, same utility despite different bundle composition]. Participants were presented with choice options that contained one fatty and one sugary milkshake with specific amounts. We estimated psychophysical IP at which a reference bundle and a variable bundle were chosen with equal probability. From these IPs, we estimated well-ordered and non-overlapping ICs. Using two independent general linear models (GLM), we found that scalar BOLD responses in striatum, midbrain and medial orbitofrontal cortex (mid-OFC) followed the IC scheme: the responses varied monotonically across ICs but changed only significantly along individual ICs, indicating orderly integration of multicomponent choice options into single-dimensional measures. The behavioral results of this study have been published in detail (Pastor-Bernier et al., 2020).

\section{Materials and Methods}

\section{Participants}

A total of 24 participants (19-36 years old with mean age 25.4 years; 11 males, 13 females) performed a binary choice task that was followed, in $50 \%$ of trials, by a Becker-DeGroot-Marschak (BDM) task inside the fMRI scanner using sugary and fatty milkshakes. All participants had known milkshake appetite, and none had diabetes or lactose intolerance. All participants provided written consent based on an information sheet. The Cambridgeshire Health Authority (Local Research Ethics
Committee) approved this study. The behavioral results have been published with more details separately (Pastor-Bernier et al., 2020).

\section{Experimental design}

The fundamental notion underlying this experiment posits that choice options consist of at least two components, and that preferences are revealed by observable choice. The multicomponent choice options are called bundles. It is immaterial for the general concept of multicomponent choice whether the individual components are parts of a single object (like size and taste of a steak in the example above) or constitute separate objects within a choice option (like lasagna and salad). Decision makers prefer bundles with larger or better components to those with smaller or worse components. Importantly, however, their preferences concern all components and are not directed at a single component alone. This property is manifested when participants prefer bundles in which one of the components of the preferred bundle is smaller than the same component in the non-preferred bundle (and the other component is large enough to overcompensate). At one point, participants may express equal preference for bundles in which the lower amount of one component is fully compensated by the higher amount in the other component, leading to choice indifference. We repeatedly measured choices with two options, each of which contained two milkshake components; the milkshakes constituted rewards, as shown by the voluntary consumption in all participants.

\section{Stimuli and rewards}

In each of the two bundles, we used stimuli to show the two milkshake components and their payout amounts (Fig. $1 A$ ). In each bundle stimulus, there were two rectangles aligned vertically. Each bundle component was indicated by the color of each rectangle. We extensively piloted various liquidized foods and liquids, and we found that milkshakes with a controlled mixture of fat and sugar give the most reliable across-participant behavioral performance. The presently used milkshakes with sugar and fat components that were found in previous studies to elicit subjective valuations and activate neural reward structures (Grabenhorst et al., 2010; Zangemeister et al., 2016). We delivered the milkshakes separately with a 0.5 -s interval (see below). As drinks consisting of only sugar or only fat were considered as too unnatural, we used a high-fat low-sugar milkshake ( $75 \%$ double cream and $25 \%$ whole milk, with no sugar) as component A (top, blue), and a high-sugar low-fat milkshake (skimmed milk with $10 \%$ sugar) as component B (bottom, red). Inside each rectangle, the vertical position of a bar indicated the component's physical amount (higher was more). We delivered the milkshakes to the participants using a custom-made silicone tubing syringe pump system (VWR International Ltd). The pump was approved for delivering foodstuffs and was controlled by a National Instruments device (NI-USB-6009) via the Data Acquisition Toolbox in MATLAB. We displayed stimuli to participants and recorded behavioral choices using the Psychtoolbox in MATLAB running on a Windows (Dell) computer (Pastor-Bernier et al., 2020).

\section{Binary choice task before fMRI scanning}

In the binary choice task, each participant revealed one's preference in repeated choices between two bundle stimuli, each indicating the amounts of two milkshake components (Fig. 1A). The two bundles (stimuli) appeared on a computer screen simultaneously in front of the participant. The left and right positions of the bundles were fixed but pseudorandomly alternated. Each bundle stimulus included the same two kinds of milkshakes with independent physical amounts. Both stimuli appeared after a pseudorandomly varying interval (mean $0.5 \mathrm{~s}$ ) after a central fixation cross. In each trial, the participant chose between the two bundles by pressing a button once (on a computer keyboard; left or right arrow corresponding to choosing left or right bundle). We defined reaction time as the interval between appearance of the two bundle stimuli and the participant's button press. We delivered the two milkshakes to the participant from the chosen bundle with a probability $p=0.2$ using a Poisson distribution; i.e., the milkshake combination of one out of an average of five chosen bundles was delivered, and no milkshake was delivered in the remaining trials. Component B (high-sugar low-fat 
milkshake) was delivered at a constant interval of $0.5 \mathrm{~s}$ after component A (high-fat low-sugar milkshake). We used this constant delay, instead of simultaneous delivery of two milkshakes or a pseudo-randomly alternating milkshake sequence, to prevent uncontrolled milkshake interactions, to maintain distinguishability of the individual milkshake rewards and to keep temporal discounting constant. Therefore, the utility of component $B$ derived from both milkshake rewards and the temporal discounting specific for each milkshake. While the interval of $0.5 \mathrm{~s}$ was sufficiently short to not disrupt task performance and data collection, it was too short to completely prevent the high-fat milkshake blending into the subsequent high-sugar milkshake inside the participant's mouth. As the interval was kept constant in all participants and at all times, the mixture provided a constant gustatory experience. Participants were asked not to eat or drink anything at least $4 \mathrm{~h}$ before the task performance. However, satiety may still be a concern given the high fat and sugar content of our milkshakes. To address this issue, we set the probability of $p=0.2$ payout schedule, limited each payout to $10.0 \mathrm{ml}$ at most, and delivered no more than a total of $200 \mathrm{ml}$ of liquid to the participant in a session. We addressed the issue with additional analyses and failed to find differential, sensory-specific satiety noticeable in choice probability measures (see below; Pastor-Bernier et al., 2020).

\section{Psychophysical assessment of IPs}

We used a psychophysical staircase method (Green and Swets, 1966; Pastor-Bernier et al., 2020) to estimate the IPs at which, by definition, each of the two bundle options was chosen equally frequently (i.e., probability $p=0.5$ for each option), indicating choice indifference for the options. We established bundles at 15 IPs for each participant and used them in the subsequent fMRI experiment.

To start the psychophysical procedure, we first set component A to $0 \mathrm{ml}$ and component $B$ to either 2, 5, or $8 \mathrm{ml}$ in the reference bundle. We then systematically varied the variable bundle. In the variable bundle, we first set the amount of its component $\mathrm{A}$ to one unit higher (mostly $0.5,1.0$, or $2.0 \mathrm{ml}$ ), we thereby specified the amount of component A gained by each participant from the choice. We then randomly selected (without replacement) one amount of component B from a total of seven fixed amounts (multiples of $0.5 \mathrm{ml}$ ), which span the whole, constant range of amounts being tested. We repeatedly selected the amounts until we tested each of the seven amounts once. We repeated estimation for each IP six times using a sigmoid function (see Eqs. 1, 1a below), requiring a total of 42 choices for estimating each IP. The amount of component B in the variable bundle was usually lower than the one in the reference bundle at the IP. With these procedures, we assessed how much of component $B$ a participant was willing to trade-in for an additional unit of component A.

We obtained more IPs from the participants' choices between the fixed reference bundle and the variable bundle, in which the amount of

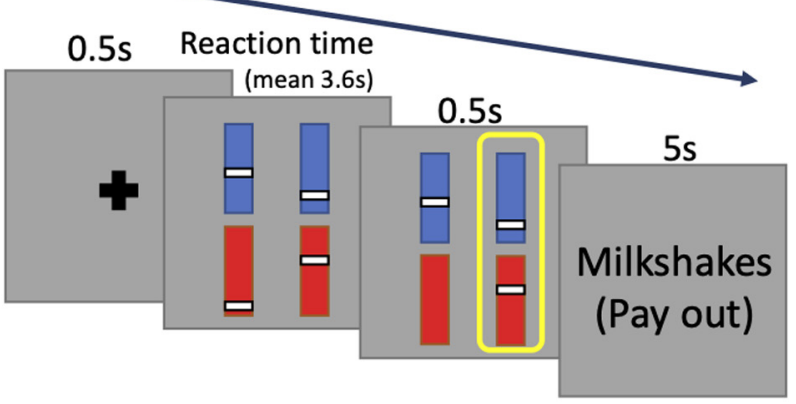

B
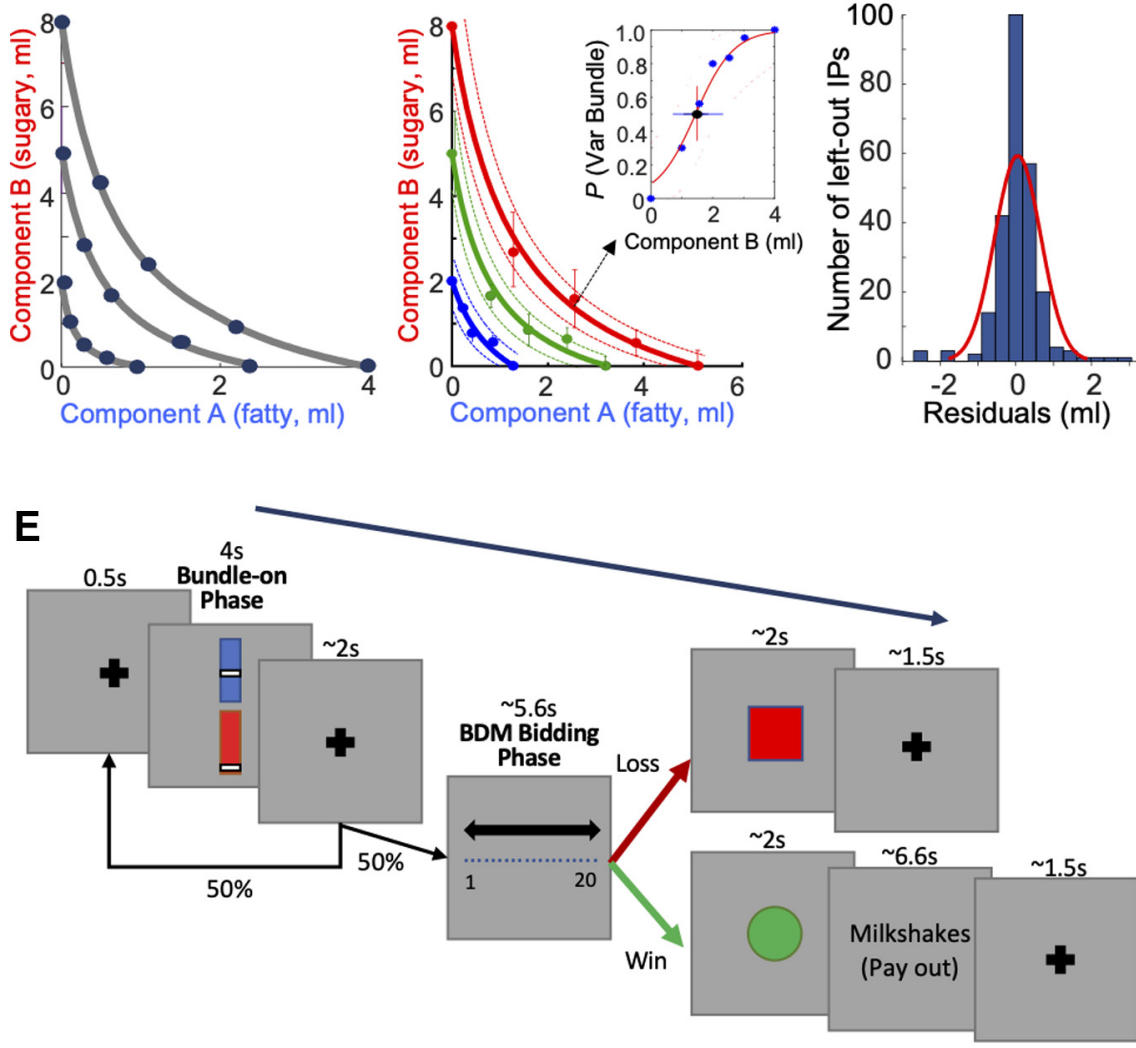

Figure 1. Experimental procedure and behavior. $\boldsymbol{A}$, Choice task outside the fMRI scanner. The participant chose between a reference bundle and a varied test bundle. Each bundle consisted of two components, component A (blue bar) and component $B$ (red bar). The amount of each component was indicated to the participant by the height of a white bar (higher was more). Component A was a low-sugar, high-fat milkshake. Component B was a low-fat high-sugar milkshake. The two milkshakes of the chosen bundle were delivered at the end of each trial with a probability of $p=0.2$. $\boldsymbol{B}$, Schematic diagram of three ICS and five IPs on each IC (same data points as shown in Pastor-Bernier et al., 2020; their Fig. 1F). C, Example ICS from a typical participant. Solid lines represent three ICs (hyperbolically fitted by IPs). Dotted lines represent $95 \%$ confidence interval of the hyperbolic fit. The inset shows the psychophysical function of one IP. The IP (black dot in the inset) was estimated by probit regression on the test points (blue dot in the inset). The same graph is shown as in Pastor-Bernier et al. (2020; their Fig. 2A). D , Histogram of residuals between fitted ICs (with a leave-one-out procedure) and left-out IPs across all participants. The residuals formed a normal symmetric distribution (red line). $\boldsymbol{E}$, Bundle task inside the fMRI scanner. At $4 \mathrm{~s}$ after the bundle-on phase, the participant performed in pseudorandomly selected $50 \%$ of trials an additional BDM task against the computer (bidding 1-20 United Kingdom pence). The reward was given if the participant won the BDM (bid $\geq$ computer bid). component A was increased stepwise, at each step varying the amount of component $\mathrm{B}$ to estimate the choice IP at which the animal was indifferent between the two bundles. Thus, bundle position advanced from top left to bottom right on the two-dimensional IC (Fig. 1B). We are aware that testing with unidirectional progression may cause particular variations in IP estimations than testing in a random sequence or in opposite directions (Knetsch, 1989). However, our primary interest in this study was to investigate basic neural processes in close relation to 
unequivocally estimated IPs and ICs rather than addressing the more advanced features of irreversibility or hysteris in ICs.

We used three different fixed amounts of component B for the reference bundle $(2,5$, or $8 \mathrm{ml})$, to obtain three IC levels. We estimated four IPs, together with the fixed reference bundle as an IP, at each of three ICs (i.e., revealed preference levels), resulting in 15 IPs, in a total of 504 choices (trials) among 84 different choice option sets in each participant (six repetitions for seven psychophysical amounts at each of the 12 IPs).

\section{Statistical analysis}

Numeric estimation of IPs

We used a sigmoid fit to numerically estimate the choice IPs. The fit was obtained from the systematically tested choices with a generalized linear regression. The generalized linear regressions used the glmfit function in MATLAB (MATLAB version R2015b) with a binomial distributed probit model, which is an inversed cumulative distribution function $(G)$. More specifically, we apply the link function to the generalized linear regression $\mathrm{y}=\beta_{0}+\beta_{1} \mathrm{~B}_{\mathrm{var}}+\varepsilon$ and write it as:

$$
\mathrm{G}(\mathrm{y})=\beta_{0}+\beta_{1} \mathrm{~B}_{\mathrm{var}}+\varepsilon,
$$

where y represents the number of trials the variable bundle is chosen in each block of a six-repetition series, $\beta_{0}$ represent the constant offset, $\beta_{1}$ represent the regression slope coefficient, $B_{\mathrm{var}}$ represent the physical reward amount $(\mathrm{ml})$ of component $\mathrm{B}$ in the variable bundle, and $\varepsilon$ represent the residual error. We used the probit model as it assumes a multivariate normal distribution of the random errors, which makes the model attractive because the normal distribution gives a good approximation to most of the variables. The model does not hypothesize error independence and is frequently used in econometrics (Razzaghi, 2013). On the other hand, the logit model, which is also commonly used in economics, is simpler to compute but has more restrictive hypotheses on error independence. Our preliminary data had shown a similar fit for both the logit and probit model, therefore, we used the probit model fit because of its less restrictive hypotheses. Thus, we approximated the IPs with the probit-model sigmoid fit, which can be written as follows:

$$
\text { Indifference Point }=-\left(\beta_{0} / \beta_{1}\right) \text {, }
$$

where $\beta_{0}$ and $\beta_{1}$ represent coefficients of the generalized linear regression (Eq. 1). We obtained these coefficients from the probit analysis (Amemiya, 1981).

ICs. In each participant, we obtained each single IC separately from an individual set of five equally revealed preferred IPs with differently composed bundles using a weighted least-square nonlinear regression. We used a weighted regression to account for choice variability within participant; the weight was defined as the inverse of the standard deviation of the titrated physical amount of component $\mathrm{B}$ at the corresponding IP (the IP having been estimated with the probit regression). We estimated the best $\beta$ coefficients from the least-square regression to obtain a single IC (utility level), using the basic hyperbolic equation:

$$
\mathrm{IC}=\beta_{0}+\beta_{1} \mathrm{~B}+\beta_{2} \mathrm{~A}+\beta_{3} \mathrm{BA}+\varepsilon,
$$

where A and B represent physical amounts of component A and component $\mathrm{B}(\mathrm{ml})$, which refer to the $x$ - and $y$-axes, respectively. Note that $(\beta 2 / \beta 1)$ is the slope coefficient and $\beta_{3}$ is the curvature coefficient of the nonlinear least-square regression. As IC is a constant (representing one utility level), we merged the IC constant with the offset constant $\left(\beta_{0}\right)$ and the error constant $(\varepsilon)$ into a common constant k. To draw the ICs, we calculated the amount of component B from the derived equation as a function of the amount of component $\mathrm{A}$ :

$$
\mathrm{B}=\left(\mathrm{k}-\beta_{2} \mathrm{~A}\right) /\left(\beta_{1}+\beta_{3} \mathrm{~A}\right) .
$$

We graphically displayed the fitted ICs (Fig. $1 B, C$ ) by plotting the preset physical amount of component $\mathrm{A}$ as the $x$ coordinates and calculated the fitted amount of component B, based on Equation 2a, as the $y$ coordinates. We estimated the error of the hyperbolic fit as the 95\% confidence interval. When calculating the ICs, we gave less weight to the IP with higher error. This model offered good fits in our earlier work (Pastor-Bernier et al., 2017, 2019, 2020). In this way, five IPs aligned to a single fitted IC. For each participant, we fitted three ICs representing increasing revealed preference levels (low, medium, high) farther away from the origin (Fig. 1B,C). The indifference map that resulted from the $3 \times 5$ IPs was unique for each of the 24 participants. The indifference maps of the 24 participants were presented before (PastorBernier et al., 2020).

\section{Leave-one-out validation of ICs}

We used a leave-one-out analysis to test the validity of the hyperbolic IC fit to the IPs. We systemically removed one IP in each IC (excluding the initial reference bundle at $\mathrm{x}=0$ ), and then fitted the IC again using the hyperbolic model. We then assessed the differences (deviation) between the original IC (without IP removal) and the new IC without the one left-out IP. The deviation was defined as the Euclidean distance of component B between the original (left-out) IP and the IP estimated from the refitted IC:

$$
\mathrm{d}=\mathrm{B}_{\mathrm{IP}}-\mathrm{B}_{\text {refit }}
$$

with $\mathrm{d}$ representing the difference (i.e., residual; in $\mathrm{ml}$; $y$-axis), $\mathrm{B}_{\mathrm{IP}}$ representing the physical amount of component B in the left-out IP (ml), and $\mathrm{B}_{\text {refit }}$ representing the estimated physical amount of component $\mathrm{B}$ in the refitted IC (ml). In this way a residual of $0 \mathrm{ml}$ suggested that removal of the left-out IP did not change the shape of that IC, while any residual unequal to $0 \mathrm{ml}$ could quantify the deviation.

\section{Control of alternative choice factors}

To assess the potential influence of other factors affecting the participants' choice, we performed a logistic regression fit on choices to test whether the choices were indeed explained by the bundle components. We performed a random-effect logistic regression on the choice data from each participant as follows:

$$
\begin{gathered}
\mathrm{y}=\beta_{0}+\beta_{1} \operatorname{RefB}+\beta_{2} \operatorname{VarA}+\beta_{3} \operatorname{VarB}+\beta_{4} \mathrm{RT}+\beta_{5} \operatorname{VarPos} \\
+\beta_{6} \text { PChoice }+\varepsilon,
\end{gathered}
$$

with y as a dummy variable (either 1 or 0 , indicating choosing or not choosing the variable bundle), RefB as physical amount ( $\mathrm{ml}$ ) of component B in the reference bundle, VarA and VarB as physical amount (ml) of components A and B in the variable bundle, RT as reaction time (ms), VarPos indicating left or right position ( 0 or 1 ) of the variable bundle stimuli shown on the computer screen relative to the reference bundle, and PChoice representing choice of the previous trial ( 0 or 1 ). Each $\beta$ coefficient was normalized by multiplying the standard deviation of the respective independent variable and dividing by the standard deviation of the dependent variable (y). We subsequently used a one-sample $t$ test against 0 to assess the statistical significance of each of the $\beta$ coefficients.

We assessed the normalized $\beta$ coefficients and $p$ values for each individual participant and then calculated averages across 24 participants. With the regression model, we found a negative correlation of choosing the variable bundle and the amount of component $\mathrm{B}$ in the reference bundle (RefB: $\beta=-0.43 \pm 0.16, p=0.020 \pm 0.005$; mean \pm SEM; amount of component A in the reference bundle was always a constant $0 \mathrm{ml}$ ). We also found positive correlation of choosing the variable bundle and amount of both component $\mathrm{A}$ and component $\mathrm{B}$ in the variable bundle (VarA: $\beta=0.67 \pm 0.16, p=0.009 \pm 0.004$; VarB: $\beta=$ $0.94 \pm 0.33, p=0.012 \pm 0.009)$. We further found that for these three variables, the $\beta$ coefficients significantly differed from 0 with one-sample $t$ tests ( $p=0.012, p=0.00088$, and $p=0.00028$, respectively), confirming the robustness of these $\beta$. Thus, we confirmed that the choices depended on the amount of reward of both variable and reference bundle. We also validated that both bundle components were important for 
the choices. All remaining variables in the regression, including reaction time, left or right position of the reference bundle on the computer screen and choice of the previous trial, failed to account significantly for the participant's current choice $(p=0.754-0.988 \pm 0.003-0.290)$. We therefore conclude that, in our experiment, the bundles with their two components, instead of other factors, account for the revealed preference relationships.

\section{Satiety control}

Besides considering other components in the design, we also tested potential effects of satiety. Satiety may have affected the preferences for the two bundle components, even if the rewards were paid out only in one fifth of the trials on average and were limited to $<200 \mathrm{ml}$. Differences in devaluation between the two-component milkshake might be a major factor for changing in an uncontrolled manner the currency relationship of the two components. This kind of unequal devaluation should result in a graded change in the instantaneous choice probability around the IPs over the test steps of 42 trials. We used the following equation to calculate the instantaneous choice probability:

$$
\mathrm{y}=\sum(\mathrm{n}=1 \text { to } 6)(\mathrm{CV} / \mathrm{TS})
$$

with y representing the instantaneous probability ( $p$ ranging from 0.0 to 1.0 ), CV represent choice or not-choice of variable bundle ( 1 or 0$)$, and TS represent test step (repetition 1-6).

We found only insignificant fluctuations in choice probabilities, without any consistent upward or downward trend in the one-way repeated measures ANOVA, together with the post hoc Tukey's test (above IP: $F_{(5,41)}=0.28, p>0.05$; below IP: $F_{(5,41)}=1.53, p>0.05$ ).

\section{Behavioral task during fMRI scanning}

During scanning, we used a value elicitation task that allowed more trials in a shorter time frame. At the beginning of each trial, one bundle was shown to the participant for $5 \mathrm{~s}$ (bundle-on phase in Fig. 1E) in the center of the computer monitor after the initial fixation period $(500 \mathrm{~ms})$. The bundle was pseudorandomly selected from the 15 IP bundles in three ICs of each participant. Bundle composition (amounts of the two components) was set in each participant according to performance in the binary choice task before fMRI scanning. Hence, the 15 bundles for each participant were not identical across participants. Subsequently, a fixation cross appeared for a pseudorandomly varying interval (mean $2 \mathrm{~s}$ ). In $50 \%$ of the trials (pseudorandomly selected), the task was terminated after this fixation cross.

In the other $50 \%$ of the trials, we presented the participant with a BDM task that was akin to a second price auction (Becker et al., 1964). This task served as an independent mechanism that related the estimated ICs to stated utility. In the BDM (bidding phase in Fig. $1 E$ ), we gave the participant a fresh 20 United Kingdom pence endowment on each trial. Using this endowment, the participant bid for a two-component bundle against a pseudorandom computer bid (extracted from a normal distribution with replacement). To bid, the participant moved a cursor, shown on the computer screen, horizontally with the left and right keyboard arrows. We registered the BDM bid (position of the cursor) $5 \mathrm{~s}$ after presenting the bidding scale to the participant. When bidding no less than the computer, the participant received the bundle (milkshake) reward from both components and paid the monetary value equal to the computer bid. By contrast, when bidding less than the computer, the participant lost the auction, paid nothing and would not get any bundle (milkshake) reward. We showed the participant the result of the auction immediately after having placed the bid, by displaying a respective win (green circle) or loss (red square) stimulus on the computer monitor (Fig. 1E); when winning the bid, the participant received the milkshake rewards in the sequence and frequency as in the binary choice task.

We first selected one bundle randomly (without replacement) from the participant-specific set of 15 bundles (the 15 bundle IPs used to fit the three ICs as shown in Fig. 1). Then we showed the participant the selected single bundle during the bundle-on phase. We presented each of the 15 bundles to the participant for 24 times, resulting in a total of
360 trials, which included 180 trials (50\%) with BDM bidding (Fig. 1E), and we used the average of these bids as the participant's BDM-estimated utility.

First, we assessed whether the BDM bids increased for bundles across revealed preference levels but were similar for IP bundles on the same revealed preference level, using Spearman's rank correlation analysis and further confirmation with the Wilcoxon signed-rank test (note that this analysis used the coordinates of the individual IPs to which the ICs had been fitted, not the IC coordinates themselves). We also performed a generalized linear regression with a Gaussian link function (randomeffect analysis) for each participant and then averaged the $\beta$ coefficients and $p$ values across all participants. We used the following generalized linear regression:

$$
\begin{aligned}
\mathrm{y}=\beta_{0} & +\beta_{1} \text { PrefLev }+\beta_{2} \text { AmBundle }+\beta_{3} \text { TrialN } \\
& +\beta_{4} \text { PrevBid }+\beta_{5} \text { Consum }+\varepsilon,
\end{aligned}
$$

with y representing amount of monetary bid, PrefLev representing revealed preference level (low, medium, high), AmBundle representing the summed amount $(\mathrm{ml})$ of component $A$ and component $B$ in the currency of component $A$ (converted with Eq. $2 \mathrm{a}$ ), TrialN representing trial number, PrevBid representing amount of monetary bid in the previous trial, and Consum representing accumulated consumption amount (ml) of component $\mathrm{A}$ and component $\mathrm{B}$ until that point in the experiment. We normalized each $\beta$ coefficient by multiplying the standard deviation of the respective independent variable, and then dividing by the standard deviation of the dependent variable $y$. We performed a subsequent onesample $t$ test against 0 to assess the significance of each $\beta$ coefficient across all 24 participants. We found significant $\beta$ coefficients of BDM monetary bids to the preference level (PrefLev: $\beta$ coefficient difference from 0: $p=0.000026$ with one-sample $t$ test; mean across all 24 participants: $\beta=0.47 \pm 0.09, p=0.016 \pm 0.015$; mean \pm SEM) and bundle amount (AmBundle: $p=0.0278 ; \beta=0.15 \pm 0.13 ; p=0.020 \pm 0.017$ ), but not in trial number (TrialN: $\beta=-0.10 \pm 0.25 ; p=0.726 \pm 0.354$ ), previous trial bid (PrevBid: $\beta=0.12 \pm 0.11 ; p=0.676 \pm 0.427$ ) nor consumption history (Consum: $\beta=0.12 \pm 0.11 ; p=0.224 \pm 0.185$ ).

\section{fMRI data acquisition}

The functional neuroimaging data in this study were collected using a 3T Siemens Magnetom Skyra Scanner at the Wolfson Brain Imaging Center. Echo-planar images (T2-weighted) with BOLD contrast were acquired at 3 Tesla across $2 \mathrm{~d}$ with each participant. All images were in plane resolution $3 \times 3 \times 2 \mathrm{~mm}$, 56 slices were acquired with 2 -mm slice thinness, repetition time $(\mathrm{TR})=3 \mathrm{~s}$, echo time $(\mathrm{TE})=30 \mathrm{~ms},-90^{\circ}$ flip angle and -192-mm field of view. To reduce signal dropout in medialtemporal and inferior-frontal regions during the scanning, the acquisition plane was tilted by $-30^{\circ}$ and the $\mathrm{z}$-shim gradient prepulse was implemented. We also applied MPRAGE sequences and co-registered to acquired high-resolution T1 structural scans for group-level anatomic localization with $1 \times 1 \times 1 \mathrm{~mm}^{3}$ voxel resolution, slice thickness of 1 $\mathrm{mm}, 2.3$-s TR, 2.98-ms TE, $9^{\circ}$ flip angle and 900-ms inversion time.

\section{fMRI data analysis}

We used the Statistical Parametric Mapping package to analyze the neuroimaging data (SPM 12; Wellcome Trust Center for Neuroimaging). We preprocessed the data by realigning the functional data to include motion correction, normalizing to the standard Montreal Neurologic Institute (MNI) coordinate, and then smoothing using a Gaussian kernel with the full width at half maximum (FWHM) of $6 \mathrm{~mm}$ within data collected on the same day. We then segmented the data to extract white matter, gray matter and CSF and followed by co-registering the 2-d data using the T1-weighted structural scans from each day. We then applied a high-pass temporal filter to it with a $128 \mathrm{~s}$ cutoff period. We applied GLMs, which assumed first-order autoregressions, to the time course of activation. We modeled event onsets, in the time course of activation, as single impulse response functions convolved with the canonical hemodynamic response. We included the time derivatives in the functions set and defined linear contrasts of parameter estimates to test the specific 
effect in each participant's dataset. We obtained voxel values for each contrast in the format of a statistical parametric map with corresponding t-statistic. We applied a standard explicit mask (mask_ICV.nii) at the first level analysis to mask out all activations outside of the brain. To test our specific hypotheses, we used the following GLMs:

\section{GLM1}

This GLM served to search for regions whose stimulus-induced brain activations varied across ICs (high $>$ low) but not along the same ICs in the bundle-stimulus-on phase (two-level $t$ test analysis; Fig. 2). For each participant, we estimated a GLM with the following regressors (R) of interest: (R1-R15) as indicator functions for each condition during the bundle-on phase (for the 15 different bundles), at the time when participant was presented with the visual bundle cue representing the milkshakes bundles; (R16) as indicator function for the BDM bid, at the time when the participant made the bid; (R17) as R16 that was modulated by the response to the participant's bid (1-20); (R18) as indicator function for the losing bid, at the time when the participant was presented with visual cues showing the loss of bidding of the trial; (R19) as indicator function for the auction win phase, at the time when the participant was presented with the visual cues representing the winning of bidding; (R20) as indicator function for the reward phase, i.e., the times when participants received the milkshakes; (R21) as R20 that was modulated by reward magnitude (in ml). Regressors R16-R19 were not used further for this analysis and served only to regress out potential BDM effects in the $50 \%$ of trials that included BDM.

In the second (group random-effects) level analysis, we entered the all 24 participant-specific linear contrasts of the first-level regressors R1R15 (representing five bundles on each of the three preference levels) into $t$ tests (high $>$ low revealed preference level) using Flexible Factorial Design, resulting in group-level statistical parametric maps. In the Flexible Factorial design matrix (second-level analysis), the following second-level regressors were used: (R1-R24) indicator functions of participant's identifier representing participants 1-24 (within participant effect); (R25-R27) indicator functions of the three revealed preference levels (across ICs; R28-R32), indicator functions of the five bundles representing amount of Component $\mathrm{A}$ in increasing magnitude or amount of component B in decreasing magnitude (along the same ICs). We first calculated the main contrast image based on high $>$ low revealed preference level ( $t$ tests). Second, we calculated a mask contrast based on five bundles of component A in increasing magnitude ( $t$ tests). Third, we calculated another mask contrast based on five bundles of component B in increasing magnitude ( $t$ tests). The final result of GLM1 was represented by the main contrast (high $>$ low revealed preference level) masking out (with exclusive mask) the two mask contrasts, controlling of the brain responses along the same ICs.

\section{GLM2}

This GLM identified regions associated with the binary comparisons of partial physical non-dominance bundles (Fig. 3). The GLM searched for brain regions in which activations were higher for bundles that were on a higher revealed preference level than bundles in which one component was physically higher than in the preferred bundle (partial physical nondominance). In the first-level estimation, regressors were the same as in GLM1 with the 21 regressors described above. In the second-level analysis, we entered all pairs of bundles that met the following criteria: (bundle 1): partial physical non-dominance bundles with higher revealed preference level, but less (with at least $0.2 \mathrm{ml}$ less in components $\mathrm{A}$ or 0.4 $\mathrm{ml}$ less in component B) in one component; (bundle 2): partial physical dominance bundles with lower revealed preference level, but more in one component. A third level group-level analysis (one-sample $t$ test) was performed with contrast images from the second level to generate group-level statistical parametric maps across 24 participants.

\section{GLM3}

This GLM identified brain regions in which activity correlated with the amount of BDM bid (0-20 pence) during the bidding phase (Fig. $4 B$ ). In the first-level estimation, we used the following regressors and parametric modulators: (R1) as indicator function of bundle-on phase; (R2) as
R1 modulated by amount of BDM bid; (R3) as indicator function of BDM bidding phase ( $50 \%$ of trials); (R4) as R3 modulated by amount of BDM bid; (R5) as indicator function of intertrial interval when there was no bidding phase (50\% of trials); (R6) as indicator function at onset of the loss cue, when the participant lost the BDM bidding; (R7) as indicator function at onset of the win cue, when the participant won the BDM bidding; (R8) as indicator function at onset of milkshake delivery; (R9) as R8 modulated by physical amount of milkshake; (R10) as contrast of win cue onset versus loss cue onset; (R11) as contrast of loss cue onset versus win cue onset. In the second-level analysis, a one-sample $t$ test analysis was performed with contrast images from the first level to generate group-level statistical parametric maps across 24 participants.

\section{Small volume corrections}

To derive coordinates for small-volume correction in GLM1 and GLM2, we entered the term "reward anticipation" in the Neurosynth meta-analysis database (Yarkoni et al., 2011) to obtain MNI coordinates. The meta-analysis employed a total of 92 independent studies that showed correlation of value elicitation with various brain regions. Our study used MNI coordinates of ventral striatum $[12,10,-8]$, mid-OFC [20, $46,-18]$ and midbrain $[8,-18,-14]$, obtained from this Neurosynth meta-analysis database. We used a sphere with 6-mm radius for midbrain and striatum, and $10 \mathrm{~mm}$ for OFC, following the common approach of using 6-mm radius spheres for subcortical structures and larger spheres for cortical structures (Chib et al., 2009; De Martino et al., 2009; Zangemeister et al., 2016).

We aimed at finding activity correlating with the BDM bid in GLM3. Therefore, for small volume correction analysis in GLM3, we used a MNI coordinate of dorsal striatum $[12,14,4]$ found in a previous study with BDM bidding (De Martino et al., 2009). We did not use coordinates from Neurosynth in GLM3 because datasets related to BDM or other auctions were not available in the Neurosynth database.

\section{Region of interest (ROI) analysis}

We selected significantly activated regions from brain maps established with GLM1, GLM2, or GLM3 for further ROI analysis. We extracted raw BOLD data from ROI coordinates based on group clusters, which we defined independently for each participant using a leave-one-out procedure based on the result of GLM1, GLM2 or GLM3. In the leaveone-out procedure, we re-estimated the second-level analysis 24 times, each time leaving out one participant, to define the ROI coordinates for the left-out participant. Following data extraction, we applied a highpass filter with a cut off period of $128 \mathrm{~s}$. The data were then $\mathrm{z}$-normalized, oversampled by a factor of 10 using the Whittaker-Shannon interpolation formula, and separated into trials to produce a matrix of trials against time.

A total of three ROI analyses were performed in this study. First, a Spearman's rank analysis was used to examine BOLD signals that changed across ICs but not along ICs (corresponding to GLM1 and GLM3). Second, a bar chart was used to illustrate the three revealed preference levels in different ROIs (corresponding to GLM1). Third, a bar chart was used to show activation changes between bundles with partial physical non-dominance on different revealed preference levels (corresponding to GLM2).

\section{Spearman's rank}

In the Spearman's rank analysis, we first regressed out the motion parameters (artifact) from the BOLD response with generalized linear models. Then we used the participant's residual BOLD response to generate time courses of Spearman's rank correlation (Rho) coefficients.

For GLM1, we tested the correlation between BOLD response (during the bundle-on phase) and revealed preference level (across-IC analysis). We then calculated group averages and standard errors of the mean for each time point for all participants, yielding averaged participant effect size time courses (Fig. 2C). In the along-IC analysis, we ranked the bundles along the same IC with individual participant's BOLD signal (Fig. 2D). A subsequent one-sample $t$ test against 0 served to assess the significance of the Rho coefficients across subjects. 
A
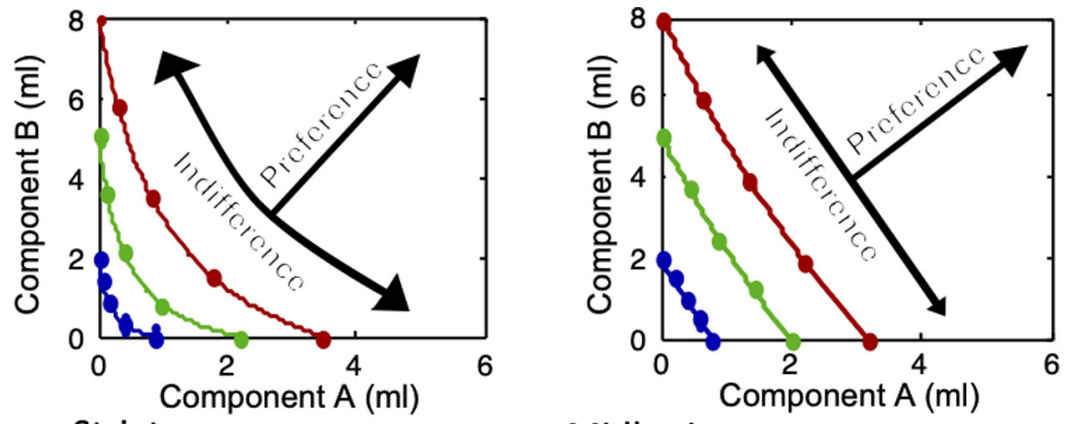

B
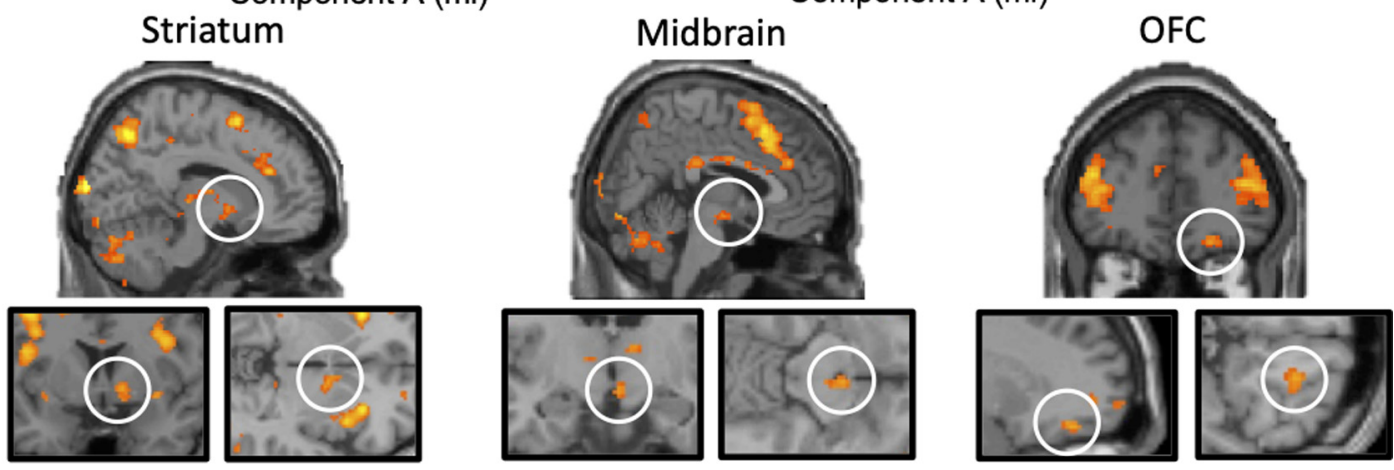

C
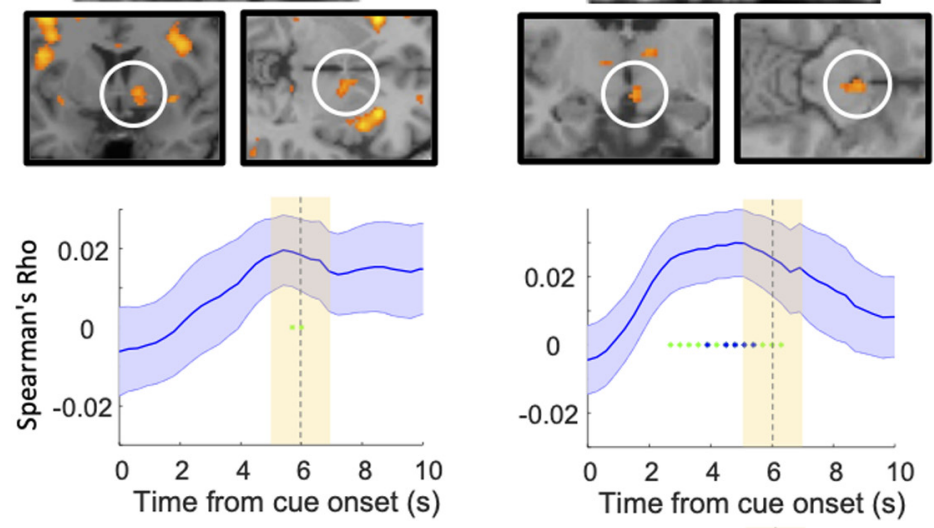

D
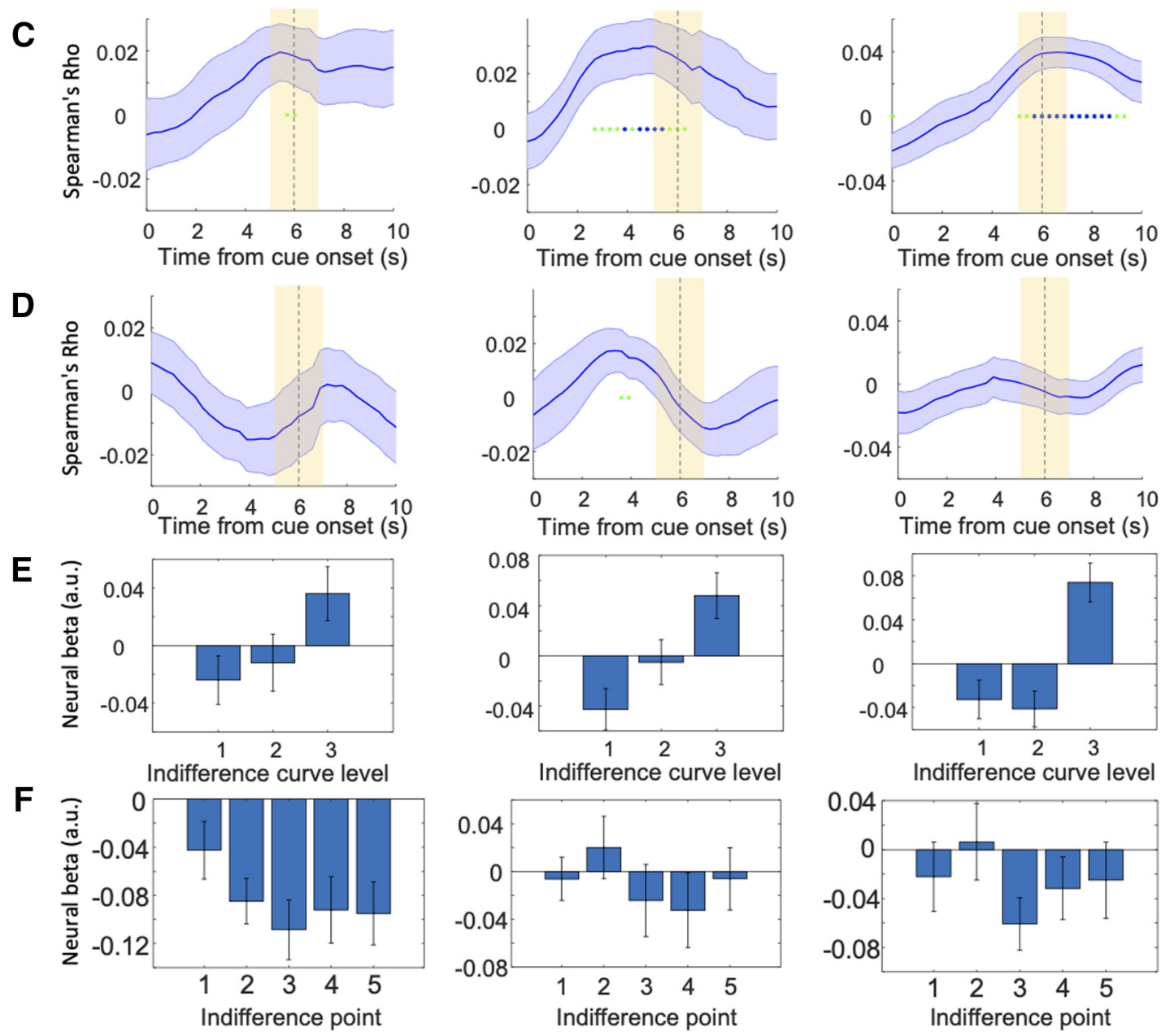

Figure 2. BOLD responses following the revealed preference scheme of two-dimensional ICS. A, Schematic of the analysis method used in GLM1 (arrows): significant BOLD signal across ICS (increasing utility) but not within ICS (same utility despite different bundle composition, as inferred from choice indifference). Participants typically showed convex ICs (left) or linear ICs (right). $B, B O L D$ responses discriminating bundles between ICS (map threshold $p<0.005$, extent threshold $\geq 10$ voxels), but no discrimination between bundles along same ICS (map threshold $p>0.005$; i.e., exclusive mask for brain response falls along the same ICs with threshold $p=0.005$ ) in a group analysis. For activations identified with $\mathrm{F}$ contrast, see Extended Data Figure 2-1. For activations identified with the lower threshold of $p<0.001$, see Extended Data Figure 2-2. C, Across-IC Spearman's rank analyses of brain activations. The Rho coefficients followed the hemodynamic response function (HRF) across the three IC levels in the ROls of the three brain structures shown above in $\boldsymbol{B}$. Solid blue lines represent mean Rho from 24 participants; 
A

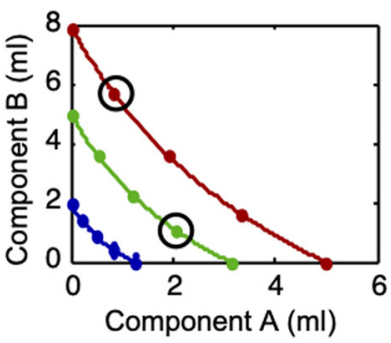

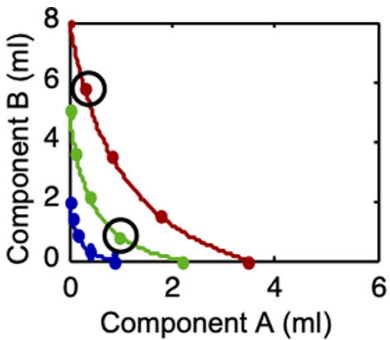

Component $\mathrm{A}(\mathrm{ml})$

B
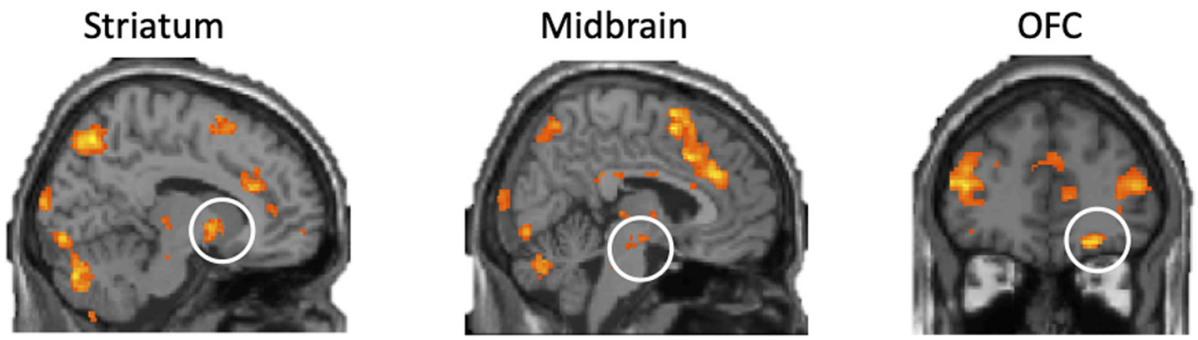

C
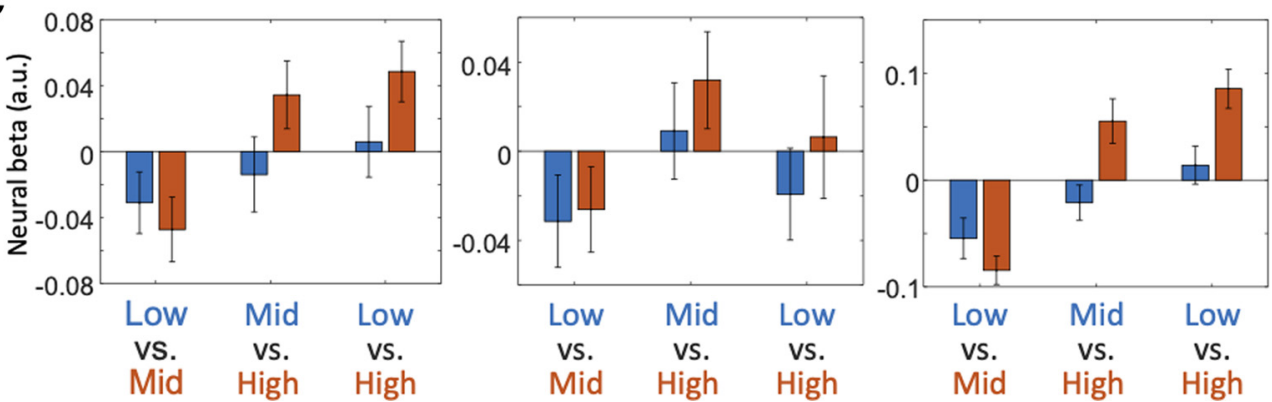

Figure 3. Higher BOLD responses to more preferred (but physically partially dominated) bundles positioned on different ICs. $\boldsymbol{A}$, Two examples of binary bundle comparison. Each pair of black circles indicates one binary comparison in one participant. $\boldsymbol{B}$, Brain regions activated more by preferred bundles compared with alternative bundles in group analysis with GLM2. Map threshold $p<0.005$, extent threshold $\geq 10$ voxels. For activations identified with the lower threshold of $p<0.001$, see Extended Data Figure 3-1. C, Bar charts showing neural $\beta$ coefficients of regression in ROls of three brain structures in the population of 24 participants. Each group of bars (three groups in each ROI) shows the $\beta$ coefficients for bundles in partial physically dominating relationships on different ICs: low versus mid, mid versus high, and low versus high. Orange bars represent the higher preference level, and blue bars represent the lower preference level in each comparison. The bars show the mean \pm SEM. For activations at peak voxels, see Extended Data Figure 3-2.

For GLM3, we tested the correlation of the BOLD response (BDM bidding phase) and the amount of BDM bids. Similar to GLM1, we then calculated group averages and standard errors of the mean of the Rho coefficients for each time point for all participants (Fig. 4B; Extended Data Fig. 4-1). A subsequent one-sample $t$ test against 0 served to assess coefficient significance.

\section{Bar chart for revealed preference level analysis}

We used bars to illustrate how different IC levels were encoded in each region of the brain. To generate an ROI bar chart, the BOLD response was first extracted using the leave-one-out procedure described above. For each participant, we obtained three generalized linear model fits to the BOLD signal at time point $6 \mathrm{~s}$. In each generalized linear model fit, the identifier of one level of revealed preference was entered as a

$\leftarrow$

\pm SEM. Yellow shaded boxes show analysis time window. Green asterisks $p<0.05$, blue asterisks $p<0.01$ for $t$ test of Spearman's Rho against zero. The BOLD responses (input of the Spearman's rank analyses; with motion parameters regressed out) were extracted from the peak voxels of each participant using with a leave-one-out procedure (see Materials and Methods). D, Along-IC ROI activations. The Spearman's rank analyses indicated hardly any significance along same ICS in ROls of the three brain structures shown in $\boldsymbol{B}$. $\boldsymbol{E}$, Bar charts of neural $\beta$ coefficients of GLM1 for the three IC levels in the three brain structures shown in $\boldsymbol{B}$ in 24 participants. Bars show mean \pm SEM. $\boldsymbol{F}$, Bar charts of neural $\beta$ coefficients of GLM1 for all five IPs on same IC levels (neural $\beta$ coefficients were averaged across the three IC levels in each participant) in 24 participants. Insignificant differences in one-way ANOVA: striatum: $p=0.3845, F_{(4,115)}=1.05$, midbrain: $p=0.6828, F_{(4,115)}=0.57 ;$ OFC: $p=0.5672$, $F_{(4,115)}=0.74$. regressor (dummy variable, e.g., 1 for bundles with high preference level and 0 for middle or low preference level) together with motion parameter regressors, which served to eliminate the motion artifact. We obtained $\beta$ coefficients of each level of revealed preference from the fit and then calculated the mean and standard error of the $\beta$ coefficient. We then plotted the bar charts shown in Figure 2E. Paired $t$ tests were used to compare $\beta$ coefficients between different revealed preference levels. As a control, we also obtained $\beta$ coefficients of five IPs from the same level of revealed preference, averaged across the three levels, and then calculated the mean and standard error of the $\beta$ coefficient across participants. We then plotted the bar charts shown in Figure $2 F$. Oneway ANOVAs were used to compare $\beta$ coefficients between the five IPs.

Bar chart for partial physical non-dominance analysis

A bundle was defined as being partially physically non-dominant over another bundle if one of its milkshake components had a physically lower amount than the same component in the dominated bundle. Thus, the revealed preferred bundle was partially physically non-dominant. For an ROI analysis of partial physical non-dominance, we fitted three generalized linear models to the BOLD response with bundle identifiers, which were two dummy variables representing partially physically dominance bundles (lower revealed preference despite larger physical amount in one milkshake) and partial physical non-dominance bundles (higher revealed preference despite smaller physical amount in one milkshake). Three generalized linear models were used to fit bundles in low versus middle, middle versus high, and low versus high comparisons, respectively. The domination was defined as at least $0.2 \mathrm{ml}$ more for component A or at least $0.4 \mathrm{ml}$ more for component $\mathrm{B}$, as in GLM2. We calculated the mean and standard error of the averaged $\beta$ coefficients 
across participants at time point $6 \mathrm{~s}$ and plotted the bar chart as shown in Figure 3C. Paired $t$ tests were used to compare $\beta$ coefficients between partial physical dominance bundles and partial physical non-dominance bundles. Motion parameters were also used as regressors for each participant to eliminate motion artefacts. In addition to extracting BOLD signal with leave-one-out peaks of GLM2 (Fig. 3C), we also extracted BOLD signal with leave-one-out peak from GLM1 (Extended Data Fig. 3-1) to confirm the robustness of this analysis.

\section{Reward prediction errors (RPEs)}

The current task did not involve learning in which reward would occur in a partly unpredicted manner and thus elicit RPEs. The only RPE could occur at the unpredicted time of the first stimulus that explicitly and quantitatively predicted the reward amounts of the bundle components indicated by the bundle stimulus. Conceivably, in the simplest form, the RPE would reflect the integrated reward amounts of both bundle components relative to the prediction derived from the past trial history. There were three levels of bundle stimulus corresponding to the three IC levels. Thus, appearance of a given bundle stimulus would elicit an RPE relative to the past experienced bundles, weighted by the learning coefficient. Thus, RPEs would have values around $-1,0$, and +1 for bundles located on low, intermediate and high ICs, respectively, the variation depending on the learning coefficient. For comparison, the bundle stimulus at each IC level without any RPE would have values of 1,2 and 3, respectively. Thus, neural responses to the RPE and to the stimulus directly (i.e., without subtraction of prediction) would result in very similar regression slopes (depending on the learning constant used for computing the RPE) and thus be difficult to distinguish from each other. We modeled RPEs with various learning coefficients in the range between 0.1 and 0.9 and for all values found high correlations between RPE and bundle stimulus value at the three IC levels. For example, a learning coefficient of 0.2 in a Rescorla-Wagner model resulted in a Spearman's rank correlation of $0.9337 \pm 0.00085$ SEM $(n=15$ bundles $\times$ 24 trials $=360$ trials $\times 24$ subjects pooled). For this reason, a RPE analysis would not yield new insights and will not be further reported.

\section{Results}

\section{Implementation of ICs}

Participants $(n=24)$ chose between two visual stimuli in repeated trials. Each of the two bundle stimuli represented a twocomponent bundle that contained the same two milkshakes with independently set amounts (Fig. 1A; see Materials and Methods). Thus, we implemented choices between bundles with separate objects (two milkshakes) rather than choices between single objects that each had multiple components. Each stimulus contained two colored vertical rectangles: the blue rectangle represented component A (low-sugar high-fat milkshake), the red rectangle represented component B (high-sugar low-fat milkshake). In each rectangle, a vertically positioned bar indicated the physical amount of each component milkshake, where higher was more.

We examined choices between: (1) a preset reference bundle and (2) a variable bundle whose component $\mathrm{A}$ had a fixed test amount and whose component B varied pseudorandomly. In all 24 participants, choice probabilities followed the component $\mathrm{B}$ monotonically. We obtained each IP (choice probability $p=0.5$ for each bundle, indicating equal preference and same utility despite different bundle composition) from a set of six-repetition choices using a probit choice function (Eqs. 1, 1a). We thereby obtained a two-dimensional IP that showed the amounts of the two components of the variable bundle between which the participant was indifferent against the constant reference bundle. We repeated this procedure, keeping the reference bundle constant and increasing the amounts of component $\mathrm{A}$ in the variable bundle, thus obtaining a set of IPs. All IPs in such a set were equally revealed preferred to, and thus had the same utility as, the constant reference bundle.

In each participant, we estimated a total of three sets of IPs (each containing five IPs) by presetting three different amounts of component $\mathrm{B}(2,5$, or $8 \mathrm{ml}$ with component $\mathrm{A}$ always $0 \mathrm{ml})$ in the reference bundle. Each IP defined the trade-off between the two components; it indicated how much of component $\mathrm{B}$ the participant was willing give up to gain one unit of component $\mathrm{A}$ without change of preference. We derived each IC from such a set of five IPs by hyperbolic fitting (Eqs. 2, 2a; Fig. 1B,C). Taken together, the IPs with the continuous ICs represented revealed preferences in a systematic manner, thus implementing the basic concepts underlying this study.

\section{Behavioral validation of $\mathrm{ICs}$}

To assess the contribution and validity of IPs (bundles) to the ICs obtained with hyperbolic fits, we performed a leave-one-out analysis. The details of these behavioral analyses were presented 
before (Pastor-Bernier et al., 2020) and are repeated here for completeness. Briefly, we left out (removed) one IP at a time from the five IPs within one fitted IC (except for the reference bundle at $\mathrm{x}=0$ ), and then we refitted the IC using the remaining four IPs with the same hyperbolic equation (see Materials and Methods; Eqs. 2, 2a). We performed the same kind of leave-oneIP-out analysis separately for each IC in each participant (four IPs on three ICs in 24 participants, resulting in 288 analyses in total).

The refitted ICs resulted in consistent fits in four measures. First, there was no overlap in the refitted IC with any refitted IC at other levels in all 24 participants; thus, the IC levels retained separation despite one IP being left-out. Second, there was no overlap in the 72 refitted ICs with the $95 \%$ confidence intervals of other original ICs at different levels; thus, the IC levels retained separation despite one IP being left-out. Third, most refitted ICs (92\%, 66 of 72 ICs) still within the 95\% confidence intervals of the original ICs without the eft-out IPs, while the remaining curves $(8 \%, 6$ of 72 ICs) showed only some parts of the IC that fell outside the $95 \%$ confidence intervals; thus, individual IPs were not overweighted in the ICs. Fourth, the left-out IPs deviated only insignificantly from the refitted ICs $(p=0.98$ with $t$ test; $N=336$; residual: $0.05 \pm 0.13 \mathrm{ml}$ in all participants, mean \pm SEM; Fig. $1 D$ ); this result confirmed that individual IPs were not overweighted in the ICs. These four validations demonstrated the robustness and consistency of the hyperbolically fitted ICs in capturing the IPs. Thus, in all participants, the ICs provided valid representations of the three revealed preference levels.

\section{Neural responses for two-component bundles across and along ICs}

During fMRI scanning, the task started with a fixation cross lasting $0.5 \mathrm{~s}$ (Fig. 1E). Then, a single two-component visual stimulus appeared in the center of the computer monitor (bundle-on phase); the stimulus predicted delivery of one of the 15 bundles (IPs) composed of two different milkshakes. The physical amount of the milkshakes in the bundle was determined by the participant-specific IP estimated from the binary choice task (see above). The participant received the two bundle milkshakes with the respective amounts indicated by the vertical bars on the stimulus, without choice. That presentation was either followed by a BDM task within the trial (50\% of trials, pseudorandomly selected) or terminated (50\% of trials). The BDM bidding served as a mechanism-independent measure of utility estimation, as used before (De Martino et al., 2013; de Berker et al., 2019). In total, each participant performed 360 trials (24 trials for each of the 15 bundles). With the fMRI data we collected, we analyzed the various aspects of neural responses (BOLD signals) to the bundles with several GLMs and ROI analyses.

We first used GLM1 to identify brain responses that follow the scheme of ICs, namely monotonic increase with higher ICs (or decrease with inverse coding) and insignificant change along the same ICs, as shown in Figure 2A. Thus, would BOLD signals change monotonically with preference and utility across ICs but vary insignificantly with choice indifference and same utility along ICs? To do so, the individual contrast images (representation of BOLD signal) of each bundle in each participant were grouped according to the IC the bundle belonged to (low, medium, high) and the position of the bundle on each IC (1-5, from top left to bottom right).

We used parametric statistical tests ( $t$ test with flexible factorial design) and estimated neuroimages of responses to each of
Table 1. Brain regions activated across but not along ICs during bundle-on phase (whole-brain analysis with GLM1)

\begin{tabular}{llcl}
\hline Brain region & Hemisphere & $\begin{array}{l}\text { MNI peak } \\
\text { coordinates }(x, y, z)\end{array}$ & $\begin{array}{l}\text { Peak } \\
z \text { score }\end{array}$ \\
\hline Striatum* & $\mathrm{R}$ & $10,6,-4$ & 3.27 \\
Midbrain* $^{*}$ & $/$ & $4,-16,-12$ & 3.09 \\
OFC* & $\mathrm{R}$ & $22,42,-16$ & 3.67 \\
Parietooccipital transition & $\mathrm{I}$ & $-12,-66,46$ & 7.42 \\
$\quad$ & & & \\
$\quad$ Insular gyrus/basal operculum & $\mathrm{L}$ & $-30,18,2$ & 5.80 \\
& $\mathrm{R}$ & $32,26,-4$ & 4.91 \\
Superior frontal gyrus & $\mathrm{I}$ & $-24,2,52$ & 5.70 \\
Middle frontal gyrus & $\mathrm{L}$ & $-42,2,42$ & 4.98 \\
& & $-40,34,18$ & 4.74 \\
& $\mathrm{R}$ & $44,46,16$ & 4.94 \\
Cingulate gyrus & $\mathrm{I}$ & $-2,-24,28$ & 4.87 \\
Precentral gyrus & $\mathrm{R}$ & $40,6,24$ & 4.59 \\
Angular gyrus & $\mathrm{L}$ & $-22,-72,54$ & 3.89 \\
\hline
\end{tabular}

Cluster $p$ values $(p<0.05)$ with family-wise error correction across the whole brain. Map threshold $p<0.005$ (across ICs; high $>$ low IC) with exclusive contrast map $p>0.005$ (along ICs), extent threshold $\geq 10$ voxels; ${ }^{*} p<0.05$ with small volume correction correction $(6-\mathrm{mm}$ radius for striatum and midbrain; $10 \mathrm{~mm}$ for $\mathrm{OFC}$ ) using coordinates from Neurosynth meta-analysis database (see Materials and Methods); / indicates activation close to and crossing the midline. For effect sizes, see Extended Data Table 1-1. For significant BOLD changes between bundles positioned on same ICS in other brain regions, see Extended Data Table 1-2.

the 15 bundles grouped into the three IC levels or five groups along ICs (see Materials and Methods). We found that the striatum, midbrain and OFC showed significantly increasing activation across increasing ICs (high $>$ low IC; map threshold of $p<0.005$; $t$ test) but insignificant variations along individual ICs (exclusive mask map threshold of $p<0.005$; Fig. $2 B$; Table 1 ; for effect sizes, see Extended Data Table 1-1). More specifically, we found small-volume corrected significance in the striatum (peak at $[10,6,-4], z$ score $=3.27,6$-mm radius sphere, cluster-level FWE corrected $p=0.041$ ), midbrain (peak at $[4,-16,-12], z$ score $=3.71,6-\mathrm{mm}$ radius sphere, cluster-level FWE corrected $p=0.048$ ) and OFC (peak at $[22,42,-16], z$ score $=3.67,10-\mathrm{mm}$ radius sphere, cluster-level FWE corrected $p=0.037$ ). (All smallvolume corrections in this study were centered on predefined coordinates from the Neurosynth meta-analysis database, see Materials and Methods). In addition, we found significant activities in other regions, including the insula and cingulate cortex (Table 1). By contrast, we found significant BOLD changes between bundles positioned on same ICs in a number of other, mostly cortical regions (Extended Data Table 1-2). These changes violated the IC scheme representing the trade-off between the two bundle rewards and were not further explored.

To provide further evidence for neural activations following the scheme of ICs, we performed a Spearman's rank time course analysis. We first extracted BOLD signals using leave-one-subject-out cross-validated GLM models, which should prevent potential biases with preselected peaks (see Materials and Methods). Subsequently we used the BOLD signals from peak voxels in each left-out subject to perform Spearman's rank analyses. We found that the striatum, midbrain and OFC showed significant Spearman's rank correlation coefficients (Spearman's Rho) between bundles located on different ICs at around $6 \mathrm{~s}$ after onset of the bundle stimulus $(p<0.05)$, consistent with the standard time course of hemodynamic response (Fig. 2C). By contrast, only insignificant $(p>0.05)$ rank coefficients were found at 5-7 s between bundles located along same ICs in these brain regions, as shown in the sliding-window analysis (Fig. 2D). These time courses followed the revealed preference to bundles across different ICs but failed to differ along the same IC, thus 
complying with the scheme of ICs that represent revealed preference. Moreover, we extracted $\beta$ (slope) coefficients of the BOLD signal at $6 \mathrm{~s}$ with the ROI coordinates identified by GLM1 and plotted them for three revealed preference levels in bar charts (Fig. 2E). We found a significant difference between high versus low revealed preference level in the midbrain $(p=0.0062)$, OFC $(p=0.0023)$, and marginal significant difference in the striatum $(p=0.0533)$. We also found a significant difference between high versus middle revealed preference level in the OFC $(p=6.8551 \times$ $10^{-4}$ ). By contrast, a one-way ANOVA analysis on the $\beta$ (slope) coefficients of the BOLD signal indicated insignificant differences between responses to five IPs positioned on same ICs in striatum, midbrain and OFC (Fig. 2F). We used F contrasts as the exclusive mask and found small volume corrected significance in striatum ( $p=0.041,6-\mathrm{mm}$ radius sphere) and OFC $(p=0.037$, 10 -mm radius sphere) but only marginal significance in midbrain ( $p=0.051,6-\mathrm{mm}$ radius sphere; Extended Data Fig. 2-1). These activations were also confirmed with the lower threshold of $p<0.001$ (Extended Data Fig. 2-2, T contrast), with small volume corrected significance in striatum $(p=0.017,6-\mathrm{mm}$ radius sphere), OFC ( $p=0.018,10-\mathrm{mm}$ radius sphere) and midbrain $(p=0.042,8-\mathrm{mm}$ radius sphere; no significance with $6 \mathrm{~mm})$.

Taken together, these data indicate that activations in several components of the brain's reward system followed the basic scheme of ICs representing revealed preferences: activation across the ICs but no activation along the same IC.

\section{Binary comparisons between partial physically non- dominant bundles}

According to the concept of ICs, any bundle on a higher IC (farther from the origin) should be preferred to any bundle on a lower IC. Hence, a single-dimensional neural signal reflecting multicomponent choice options should vary between any bundle on a higher IC and any bundle on a lower IC. To reflect the proper integration of the two bundle components regardless of specific physical properties, the neural signal should follow the IC rank even when one component milkshake of the higher-IC bundle is lower than in the lower-IC bundle (partial physical non-dominance). To identify such differences, we used the GLM2. With pairwise comparisons, GLM2 should identify higher responses to revealed preferred bundles with partial physical non-dominance. Thus, GLM2 compared all bundle pairs that fit the following condition within each participant: bundle 1 was located on higher IC but had a lower amount of one component milkshake compared with bundle 2 that was located on a lower IC (Fig. 3A).

The GLM2 analysis demonstrated significant activations in similar regions as with GLM1, where striatum (peak at $[16,6$, $-6], z$ score $=3.8,6-\mathrm{mm}$ radius sphere, cluster-level FWE corrected $p=0.012$ ), midbrain (peak at $[4,-16,-12], z$ score $=2.85$, $6-\mathrm{mm}$ radius sphere, cluster-level FWE corrected $p=0.032$ ) and OFC (peak at $[24,42,-16], z$ score $=3.99,10$ - $\mathrm{mm}$ radius sphere, cluster-level FWE corrected $p=0.012$ ) showed small-volume corrected significant activations (Fig. $3 B$ ). These activations were also confirmed with the lower threshold of $p<0.001$ (Extended Data Fig. 3-1), with small volume corrected significance in striatum $(p=0.008,6-\mathrm{mm}$ radius sphere) and OFC $(p=0.004,10-$ $\mathrm{mm}$ radius sphere). Also, we found significant activities in other regions, including insula, superior frontal gyrus and cingulate, as shown in Table 2.

We also performed ROI analyses (coordinates identified by GLM2 with leave-one-subject-out procedure) that calculated betas of partial physical non-dominance (higher revealed
Table 2. Brain regions showing differences (partial physical non-dominance $>$ partial physical dominance) in BOLD signal between partial physically dominating bundles located on different ICs during bundle-on phase (whole-brain analysis with GLM2)

\begin{tabular}{llcl}
\hline Brain region & Hemisphere & MNI peak coordinates $(x, y, z)$ & Peak z score \\
\hline Striatum* & $\mathrm{R}$ & $16,6,-6$ & 3.8 \\
Midbrain* $^{*}$ & $/$ & $4,-16,-12$ & 2.85 \\
OFC & $\mathrm{R}$ & $24,42,-16$ & 3.99 \\
Insular gyrus/basal operculum & $\mathrm{L}$ & $-30,24,-2$ & 5.55 \\
& $\mathrm{R}$ & $32,26,-6$ & 4.40 \\
Angular gyrus & $\mathrm{R}$ & $32,-68,28$ & 5.51 \\
Cerebellum & $\mathrm{L}$ & $-36,-68,-30$ & 4.79 \\
Superior frontal gyrus & $/$ & $22,2,54$ & 4.75 \\
Occipital gyri & $\mathrm{L}$ & $-28,-88,4$ & 4.57 \\
Middle frontal gyrus & $\mathrm{L}$ & $-50,40,16$ & 4.32 \\
& $\mathrm{R}$ & $46,42,14$ & 3.76 \\
Inferior frontopolar gyrus & $\mathrm{R}$ & $18,64,-8$ & 4.11 \\
Cingulate gyrus & $/$ & $-2,-24,28$ & 4.05 \\
\hline
\end{tabular}

Cluster $p$ values $(p<0.05)$ with family-wise error correction across the whole brain. Map threshold $p<0.005$, extent threshold $\geq 10$ voxels; ${ }^{*} p<0.05$ with small volume correction correction (6-mm radius for striatum and midbrain; $10 \mathrm{~mm}$ for $\mathrm{OFC}$ ) using coordinates from Neurosynth meta-analysis database (see Materials and Methods).

preference) and partial physical dominance bundles (lower revealed preference) as described in Materials and Methods. For each ROI, we computed three models, which compared bundles pairwise, with low versus middle, middle versus high, and low versus high revealed preference, respectively. Neural $\beta$ regression coefficients were extracted at $6 \mathrm{~s}$ after the onset of the bundle stimulus, which corresponded to the canonical hemodynamic response. In regard to high versus low revealed preference level, we found significance in the striatum $(p=0.0459)$ and OFC $(p=0.0033)$ when comparing bundles in high IC versus low IC (Fig. $3 C$ ). We also found significance in the striatum $(p=0.0309)$ and $\operatorname{OFC}\left(p=7.6575 \times 10^{-5}\right)$ when comparing high versus middle IC bundles. In the midbrain, we found no significance $(p>0.05)$ in the three comparisons between bundles on low, middle and high ICs (although such a tendency existed in all three comparisons). When plotting Figure $3 C$ using peak voxels from GLM1, we found similar results for all three regions (Extended Data Fig. 3-2), which is unsurprising as the coordinates were similar between GLM1 and GLM2. Thus, the ROI analysis was robust with GLM1 coordinates for these regions.

Taken together, these pairwise bundle comparisons demonstrated neural coding of partial physical non-dominance bundles as a necessary condition for extracting a scalar neural signal from vectorial, multicomponent choice options. These results confirmed compliance with the graphic schemes of ICs demonstrated with GLM1.

\section{BDM control of revealed preference}

To validate the order of revealed preferences represented by the ICs with an independent estimation mechanism, we used a monetary BDM bidding task that estimated each participant's utility for each bundle. In 50\% of trials during fMRI scanning, each participant made a monetary BDM bid (United Kingdom pence) for one of the 15 bundles, out of a fresh endowment of 20 United Kingdom pence in each trial (BDM bidding phase; Fig. $1 E$ ). The 15 bundles constituted the IPs of the ICs that were estimated during the binary choice task with each participant.

The BDM bids followed the order of revealed preference levels across ICs, as demonstrated by significant positive Spearman's rank correlation between the three IC levels and the 
bid amounts for bundles and confirmed with significant binary Wilcoxon signed-rank tests between the three IC levels (Fig. $4 A$, blue, green, red). By contrast, there was no correlation between bids for the five bundles and their position along each IC (from top left to bottom right; Spearman's Rho $=0.0219 ; p=0.6791$ ). Thus, BDM bids increased across the three IC levels but did not change monotonically with bundle position along individual ICs in the population of our participants.

In order to investigate neural mechanisms of BDM bidding and value elicitation, we compared two GLM models: (1) GLM3 to identify brain regions that encoded BDM bids (0-20 pence) during the bidding phase, as shown in Figure 4B; (2) GLM1 to identify brain regions that encoded value elicitation according to IC levels during bundle-on phase, as shown above in Figure $2 B$, $C$, far right.

Analysis with GLM3 demonstrated activation in ventromedial prefrontal cortex (vmPFC) that encoded BDM bids during the bidding phase (peak at $[6,44,0], z$ score $=4.10$, whole-brain corrected with cluster-level FWE corrected $p=0.002$; Fig. $4 B$, left), together with other brain regions (Table 3 ). Further ROI analysis showed significant rank correlation between vmPFC activation and $\mathrm{BDM}$ bids at around $6 \mathrm{~s}$ after BDM cue onset (bidding phase; $p<0.05$; Spearman's Rho; Fig. $4 B$, right), consistent with the expected hemodynamic response function. By contrast, analysis with GLM1 showed significant, small-volume corrected activation in OFC that indicated its involvement in encoding IC levels during the bundle-on phase (Fig. $2 B$, far right). The ROI analysis showed significant rank correlation between OFC activation and IC levels at around $6 \mathrm{~s}$ after bundle onset (bundle-on phase; $p<0.05$; Spearman's Rho; Fig. $2 C$, far right). In addition, with GLM3, we found small-volume corrected significant encoding of BDM bids in the dorsal striatum (peak at $[12,12,0], z$ score $=3.53,6-\mathrm{mm}$ radius sphere, cluster-level FWE corrected $p=0.008$; Extended Data Fig. $4-1)$, whereas BDM encoding was insignificant in the ventral striatum $(p>0.1)$.

Taken together, BDM bidding provided a good validation of the estimated levels of revealed preference represented by ICs. However, and interestingly, revealed preference levels and BDM bids were encoded in different regions of the frontal cortex and striatum.

\section{Discussion}

We systematically tested characteristics of scalar neural responses to vectorial, multicomponent bundles. We estimated IPs by asking human participants to choose between two bundles. Each bundle contained the same two separate objects (milkshakes) rather than consisting of single objects that each had multiple components. Our behavioral results (Pastor-Bernier et al., 2020) showed that preference relationships among multicomponent choices were reliably represented by systematic ICs, as a prerequisite for testing the underlying neural mechanisms. In fMRI scans with GLM and post hoc ROI analyses, we identified brain regions whose activations correlated with levels of revealed preference. The GLM1 and post hoc Spearman's rank analysis demonstrated activations in the ventral striatum, midbrain and OFC that reflected revealed preference levels across ICs (changing utility) but failed to vary along equal-preference ICs (same utility despite different bundle composition). The GLM2 specifically dissociated revealed preference from physical dominance and showed consistent results with those from GLM1. A mechanismindependent control with a BDM bidding task confirmed the validity of ICs for representing revealed preference levels.
Table 3. Brain regions with BOLD responses correlating with BDM bids during the bidding phase (whole-brain analysis with GLM3)

\begin{tabular}{llcl}
\hline Brain region & Hemisphere & $\begin{array}{l}\text { MNI peak } \\
\text { coordinates }(x, y, z)\end{array}$ & $\begin{array}{l}\text { Peak } \\
z \text { score }\end{array}$ \\
\hline vmPFC & $/$ & $6,44,0$ & 4.10 \\
Dorsal striatum* & $\mathrm{R}$ & $12,12,0$ & 3.53 \\
Insular gyrus/basal operculum & $\mathrm{R}$ & $30,24,-2$ & 5.21 \\
Inferior frontal gyrus, opercular part & $\mathrm{R}$ & $46,8,22$ & 4.76 \\
Occipital gyri & $\mathrm{L}$ & $-36,-90,-2$ & 4.67 \\
Superior parietal lobule & $\mathrm{L}$ & $-30,-56,46$ & 4.14 \\
Superior frontal gyrus & $/$ & $2,26,42$ & 4.05 \\
Middle frontal gyrus & $\mathrm{R}$ & $38,36,18$ & 3.93 \\
Postcentral gyrus & $\mathrm{R}$ & $32,-36,48$ & 3.62 \\
Inferior frontopolar gyrus & $\mathrm{R}$ & $22,56,-4$ & 3.53 \\
\hline
\end{tabular}

Cluster $p$ values $(p<0.05)$ with family-wise error correction across the whole brain. Map threshold $p<0.005$, extent threshold $\geq 10$ voxels; $* p<0.05$ with small volume correction (6-mm radius) using coordinates from a previous study with BDM bidding (De Martino et al., 2009; see Materials and Methods).

Interestingly, however, BDM bidding was associated with activations in vmPFC and dorsal striatum rather than the previously identified reward structures following IC levels. Together, these data demonstrate systematic, single-dimensional neural activations in the striatum, midbrain and OFC that reflect preferences for, and utility of, vectorial multicomponent choice options.

Scalar neural activations from vectorial choice options are only the simplest way to represent value integrated from multiple components. Other plausible but less straightforward ways might be ensemble coding composed of multiple heterogeneous signals representing only single components of multicomponent options, as seen in individual OFC neurons (Pastor-Bernier et al., 2019). Future neuroimaging studies may address such issues.

In our binary choice task, we elicited revealed preferences with repeated, psychophysically controlled choices (Green and Swets, 1966; Pastor-Bernier et al., 2020). Such a multitrial, stochastic approach is well conceptualized (McFadden and Richter, 1990; McFadden, 2005), fulfils statistical requirements of neural research, corresponds to standard choice functions (Sutton and Barto 1998), and allows comparison with animal neurophysiology (Pastor-Bernier et al., 2017). These methods delivered varying choice probabilities (stochastic choices) instead of single selections (deterministic choices).

Economic choice experiments often involve substantial but imaginary sizes or amounts of consumer items and money, or use random singular payouts (Simonson, 1989; Tversky and Simonson, 1993; Rieskamp et al., 2006). By contrast, our payout schedule fit the requirements of neuroimaging and involved tangible and consumable rewards over hundreds of trials, while also controlling for satiety. The behavioral choices resembled small daily activities, such as drink and snack consumption. In this way, we obtained three well-ordered ICs for each participant that provided accurate and systematic representations of preferences for multicomponent bundles, without involving imagined items or monetary reward (Pastor-Bernier et al., 2020).

We used the BDM task as an authoritative, mechanism-independent control for eliciting subjective values, thereby providing an additional validating mechanism for the revealed preferences elicited in our binary choice test. The value estimating mechanism for BDM bids differs substantially from the one for revealed preference ICs. The truthful revelations (incentive compatibility) of BDM makes this mechanism an essential tool in experimental economics that is becoming more popular in human decision research (Plassmann et al., 2007; Medic et al., 2014; 
Zangemeister et al., 2016). The elicited BDM bids correlated well with the revealed preference levels (Pastor-Bernier et al., 2020) and thereby validated in a mechanism-independent manner the empirically estimated IPs used during fMRI (in which the participants performed the BDM task). Previous neuroimaging studies showed activations in vmPFC that correlated with BDM bids (Chib et al., 2009; McNamee et al., 2013). Our experimental design dissociated value elicitation by bundles and by BDM bidding. We confirmed the BDM activations in vmPFC and found that the two mechanistically different tasks activated different regions in both PFC and striatum; responses to the bundles followed the IC scheme (different activations across but not within ICs) in OFC and ventral striatum, whereas BDM bidding activated vmPFC and dorsal striatum. Previous studies showed that vmPFC activity can reflect value derived from both rating measures and can distinguish between preferred and non-preferred options regardless of task demands (Lebreton et al., 2009; LopezPersem et al., 2020). Thus, the conditions under which vmPFC encodes value, and the precise form of value-elicitation that best explains vmPFC activity are valuable topic for future studies.

Previous studies tested neural mechanisms of human choice of bundles with multiple components, such as payoff amount and probability (Chau et al., 2014), quality and quantity of goods (de Berker et al., 2019), money and time (Gluth et al., 2017), and food components (Suzuki et al., 2017). Nevertheless, none of these studies tested bundles that were positioned along modeled ICs (i.e., eliciting choice indifference) and thus failed to test the crucial trade-off that demonstrates the graded and well-ordered manner of single-dimensional preferences for multidimensional choice options. Without this information, we would not know how a scalar neural response may arise from graded changes of vectorial, multicomponent bundles. Our study, testing five bundles on each IC, addressed this problem and identified the brain regions that showed this kind of neural response.

Although we tested the emergence of single-dimensional neural signals for multidimensional bundles in a systematic and concept-driven way, there were limitations with our experimental design. First, both bundle components had the same type of primary reward (milkshakes). It would be interesting to study whether the same brain regions would encode different types of rewards and follow the formalisms of ICs, including the graded trade-off. For instance, future research may compare monetary rewards with primary nutrient rewards. Second, we only demonstrated neural responses with the typical convex ICs. It would be interesting to study whether different brain regions might encode preferences with different shapes of ICs. Such work may test participants' choices with linear or concave ICs. Third, we did not test the influences of prior experience on current decisions. Previous studies (Schultz, 1998; van den Bos et al., 2013; LopezPersem et al., 2016) showed that choices could be influenced by previous experience and be updated by reinforcement learning. Future research may include multicomponent choice options during fMRI scanning to study multicomponent reinforcement learning. Lastly, we only demonstrated fMRI BOLD responses, and future neurophysiology research should confirm the coding of revealed preference at a single neuron level in human patients with intracerebrally implanted electrodes, similar to our recently investigated neuronal encoding of revealed preference in monkey OFC (Pastor-Bernier et al., 2019). To conclude, while we showed brain activation with bundles in a formal but standard revealed preference setting (convex ICs, primary reward), it is desirable to know how human brains encode revealed preference in a larger variety of situations.
The reward circuit including the striatum and midbrain is known to participate in reward anticipation and learning, including RPE (Diederen et al., 2017). In monkeys, midbrain dopamine neurons encode values for predicted rewards in economic decision tasks (Lak et al., 2016; Schultz et al., 2017). Similar to the midbrain and striatum, previous work showed the involvement of the human mid-OFC in valuation of primary nutrient reward (Grabenhorst et al., 2010) and monetary reward (Kahnt et al., 2014). Remarkably, the neural activity in OFC elicited here, in response to visual cues predicting liquid rewards with varying sugar and fat components, closely matched the coordinates observed previously (Grabenhorst et al., 2010) in a study in which subjects orally sampled very similar liquid rewards. Thus, this area of OFC seems to be involved both in reward valuation during oral consumption of primary nutrient rewards and in the economic valuation of visually cued choice options. In nonhuman primates, OFC neurons encode reward prediction (Tremblay and Schultz, 1999; Padoa-Schioppa and Assad, 2006) and follow revealed preferences for multicomponent bundles (Pastor-Bernier et al., 2019). In the current study, we used a concept-driven design and found that neural responses in the striatum, midbrain and OFC integrated multiple bundle components in a way that followed the ICs scheme (changing across ICs but being similar along equal-preference ICs). Moreover, we demonstrate the involvement of the midbrain in multicomponent decision-making for the first time. Overall, our results show the involvement of principal reward structures of the brain in integrating the multiple components of vectorial bundles into singledimensional neural signals that are suitable for economic decision-making.

Besides the primary reward circuit (midbrain dopamine neurons, OFC, striatum, amygdala), other brain regions are also involved in economic decision-making. Previous studies in multicomponent decision-making suggested the involvement of the cingulate, PFC, and insula in value elicitation (Kurtz-David et al., 2019; Busemeyer et al., 2019). Consistent with these studies, we also found significant activation in these regions. As shown in Tables 1, 2, the BOLD signals identified by GLM1 and GLM2 showed that these regions also encode bundle values during the bundle-on phase, together with the striatum, midbrain, and midOFC. Our results are consistent with these previous studies, suggesting that a considerable number of brain regions also play a role in multicomponent decision-making.

\section{References}

Amemiya T (1981) Qualitative response models: a survey. J Econ Litt 19:1483-1536.

Becker GM, DeGroot MH, Marschak J (1964) Measuring utility by a singleresponse sequential method. Behav Sci 9:226-232.

Busemeyer JR, Gluth S, Rieskamp J, Turner BM (2019) Cognitive and neural bases of multi-attribute, multi-alternative, value-based decisions. Trends Cogn Sci 23:251-263.

Chau BKH, Kolling N, Hunt LT, Walton ME, Rushworth MFS (2014) A neural mechanism underlying failure of optimal choice with multiple alternatives. Nat Neurosci 17:463-470.

Chib VS, Rangel A, Shimojo S, O’Doherty JP (2009) Evidence for a common representation of decision values for dissimilar goods in human ventromedial prefrontal cortex. J Neurosci 29:12315-12320.

Chung HK, Sjöström T, Lee HJ, Lu YT, Tsuo FY, Chen TS, Chang CF, Juan $\mathrm{CH}$, Kuo WJ, Huang CY (2017) Why do irrelevant alternatives matter? An fMRI-TMS study of context-dependent preferences. J Neurosci 37:11647-11661.

de Berker AO, Kurth-Nelson Z, Rutledge RB, Bestmann S, Dolan RJ (2019) Computing value from quality and quantity in human decision-making. J Neurosci 39:163-176. 
De Martino B, Kumaran D, Holt B, Dolan RJ (2009) The neurobiology of reference-dependent value computation. J Neurosci 29:3833-3842.

De Martino B, Fleming SM, Garrett N, Dolan RJ (2013) Confidence in valuebased choice. Nat Neurosci 16:105-110.

Diederen KM, Ziauddeen H, Vestergaard MD, Spencer T, Schultz W, Fletcher PC (2017) Dopamine modulates adaptive prediction error coding in the human midbrain and striatum. J Neurosci 37:1708-1720.

Fujiwara J, Tobler PN, Taira M, Iijima T, Tsutsui KI (2009) Segregated and integrated coding of reward and punishment in the cingulate cortex. J Neurophysiol 101:3284-3293.

Gluth S, Hotaling JM, Rieskamp J (2017) The attraction effect modulates reward prediction errors and intertemporal choices. J Neurosci 37:71382.

Grabenhorst F, Rolls ET, Parris BA, d'Souza AA (2010) How the brain represents the reward value of fat in the mouth. Cereb Cortex 20:1082-1091.

Green DM, Swets J (1966) Signal detection theory and psychophysics. New York: Wiley.

Hunt LT, Dolan RJ, Behrens TE (2014) Hierarchical competitions subserving multi-attribute choice. Nat Neurosci 17:1613-1620.

Kagel JH, Battalio RC, Rachlin H, Basmann RL, Green L, Klemm WR (1975) Experimental studies of consumer demand behavior using laboratory animals. Econ Inquiry 13:22-38.

Kahnt T, Park SQ, Haynes JD, Tobler PN (2014) Disentangling neural representations of value and salience in the human brain. Proc Natl Acad Sci USA 111:5000-5005.

Knetsch JL (1989) The endowment effect and evidence of nonreversible indifference curves. Am Econ Rev 79:1277-1288.

Kurtz-David V, Persitz D, Webb R, Levy DJ (2019) The neural computation of inconsistent choice behavior. Nat Comm 10:1583.

Lak A, Stauffer WR, Schultz W (2016) Dopamine neurons learn relative chosen value from probabilistic rewards. Elife 5:e18044

Lebreton M, Jorge S, Michel V, Thirion B, Pessiglione M (2009) An automatic valuation system in the human brain: evidence from functional neuroimaging. Neuron 64:431-439.

Lopez-Persem A, Domenech P, Pessiglione M (2016) How prior preferences determine decision-making frames and biases in the human brain. Elife 5:e20317.

Lopez-Persem A, Bastin J, Petton M, Abitbol R, Lehongre K, Adam C, Navarro V, Rheims S, Kahane P, Domenech P, Pessiglione M (2020) Four core properties of the human brain valuation system demonstrated in intracranial signals. Nat Neurosci 23:664- 675.

Mas-Colell A, Whinston MD, Green JR (1995) Microeconomic theory. New York: Oxford Univ Press.

McFadden DL (2005) Revealed stochastic preference: a synthesis. Econ Theory 26:245-264.

McFadden D, Richter MK (1990) Stochastic rationality and revealed stochastic preference. In: Preferences, uncertainty, and optimality. Essays in honor of Leo Hurwicz, pp 161-186. Boulder: Westview Press.

McNamee D, Rangel A, O’Doherty JP (2013) Category-dependent and category-independent goal-value codes in human ventromedial prefrontal cortex. Nat Neurosci 16:479-485.
Medic N, Ziauddeen H, Vestergaard MD, Henning E, Schultz W, Farooqi IS, Fletcher PC (2014) Dopamine modulates the neural representation of subjective value of food in hungry subjects. J Neurosci 34:16856-16864.

Padoa-Schioppa C, Assad JA (2006) Neurons in the orbitofrontal cortex encode economic value. Nature 441:223-226.

Pastor-Bernier A, Plott CR, Schultz W (2017) Monkeys choose as if maximizing utility compatible with basic principles of revealed preference theory. Proc Natl Acad Sci USA 114:E1766-E1775.

Pastor-Bernier A, Stasiak A, Schultz W (2019) Orbitofrontal signals for twocomponent choice options comply with indifference curves of revealed preference theory. Nat Commun 10:4885

Pastor-Bernier A, Volkmann K, Stasiak A, Grabenhorst F, Schultz W (2020) Experimentally revealed stochastic preferences for multi-component choice options. J exp Psychol Anim Learn Cog 46:367-384.

Plassmann H, O'Doherty J, Rangel A (2007) Orbitofrontal cortex encodes willingness to pay in everyday economic transactions. J Neurosci 27:9984-9988.

Razzaghi M (2013) The probit link function in generalized linear models for data mining applications. J Mod Appl Stat Meth 12:Article 19.

Rieskamp J, Busemeyer JR, Mellers BA (2006) Extending the bounds of rationality: evidence and theories of preferential choice. J Econ Lit 44:631-661.

Samuelson PA (1938) A note on the pure theory of consumer's behavior. Economica 5:61-71.

Schultz W (1998) Predictive reward signal of dopamine neurons. J Neurophysiol 80:1-27.

Schultz W, Stauffer WR, Lak A (2017) The phasic dopamine signal maturing: from reward via behavioural activation to formal economic utility. Curr Opin Neurobiol 43:139-148.

Simonson I (1989) Choice based on reasons: the case of attraction and compromise effects. J Consum Res 16:158-174.

Sutton RS, Barto AG (1998) Reinforcement learning: an introduction. Cambridge: MIT Press.

Suzuki S, Cross L, O’Doherty JP (2017) Elucidating the underlying components of food valuation in the human orbitofrontal cortex. Nat Neurosci 20:1780-1786

Tremblay L, Schultz W (1999) Relative reward preference in primate orbitofrontal cortex. Nature 398:704-708.

Tversky A, Simonson I (1993) Context-dependent preferences. Management Sci 39:1179-1189.

van den Bos W, Talwar A, McClure SM (2013) Neural correlates of reinforcement learning and social preferences in competitive bidding. J Neurosci 33:2137-2146

Yarkoni T, Poldrack RA, Nichols TE, Van Essen DC, Wager TD (2011) Large-scale automated synthesis of human functional neuroimaging data. Nat Methods 8:665-670.

Zangemeister L, Grabenhorst F, Schultz W (2016) Neural basis for economic saving strategies in human amygdala-prefrontal reward circuits. Curr Biol 26:3004-3013. 